\title{
Alterations of oral microbiota in Chinese children with viral encephalitis and/or viral meningitis
}

\author{
Yijie $\mathrm{Li}^{1,2 \dagger}$, Jing $\mathrm{Liu}^{3 \dagger}$, Yimin Zhu ${ }^{1,2}$, \\ Chunying Peng ${ }^{3}$, Yao Dong ${ }^{1,2}$, Lili Liu ${ }^{1,2}$, \\ Yining $\mathrm{He}^{1,2}$, Guoping $\mathrm{Lu}^{3 *}$, \\ and Yingiie Zheng ${ }^{1,2,4}$ \\ ${ }^{1}$ Department of Epidemiology, School of Public Health, \\ Fudan University, Shanghai 200032, P. R. China \\ ${ }^{2}$ Key Laboratory for Health Technology Assessment, National \\ Commission of Health and Family Planning, Fudan University, \\ Shanghai 200032, P. R. China \\ ${ }^{3}$ Pediatric Intensive Care Unit, Children's Hospital of Fudan University, \\ National Center for Children's Health, Shanghai 201102, P. R. China \\ ${ }^{4}$ Laboratory of Public Health Safety, Ministry of Education, School of \\ Public Health, Fudan University, Shanghai 200032, P. R. China \\ (Received Oct 25, 2021 / Revised Dec 15, 2021 / Accepted Jan 6, 2022)
}

The role of oral microbiota in viral encephalitis and/or viral meningitis (VEVM) remains unclear. In this hospital-based, frequency-matched study, children with clinically diagnosed VEVM $(n=68)$ and those with other diseases (controls, $n=$ 68) were recruited. Their oral swab samples were collected and the oral microbiota was profiled using 16S rRNA gene sequencing. The oral microbiota of children with VEVM exhibited different beta diversity metrics (unweighted UniFrac distance: $P<0.001, \mathrm{R}^{2}=0.025$, Bray-curtis dissimilarity: $P$ $=0.045, \mathrm{R}^{2}=0.011$, and Jaccard dissimilarity: $P<0.001, \mathrm{R}^{2}$ $=0.017)$ and higher relative abundances of taxa identified by Linear discriminant analysis (LDA) with effect size (Enterococcus, Pedobacter, Massilia, Prevotella_9, Psychrobacter, Butyricimonas, Bradyrhizobium, etc., LDA scores $>2.0$ ) when compared with the control group. The higher pathway abundance of steroid hormone biosynthesis predicted by oral microbiota was suggested to be linked to VEVM $(q=0.020)$. Further, a model based on oral microbial traits showed good predictive performance for VEVM with an area under the receiver operating characteristic curve of 0.920 (95\% confidence interval: 0.834-1.000). Similar results were also obtained between children with etiologically diagnosed VEVM $(n=43)$ and controls $(n=68)$. Our preliminary study identified VEVM-specific oral microbial traits among children, which can be effective in the diagnosis of VEVM.

Keywords: oral microbiota, viral encephalitis, viral meningitis, 16S rRNA, random forest, diagnostic performance

\footnotetext{
${ }^{\dagger}$ These authors contributed equally to this work.

*For correspondence. (Y. Zheng) E-mail: yjzheng@fudan.edu.cn; Tel.: +8613764425579 / (G. Lu) E-mail: 13788904150@163.com; Tel.: +86-137889 04150

${ }^{8}$ Supplemental material for this article may be found at

http://www.springerlink.com/content/120956.

Copyright (c) 2022, The Microbiological Society of Korea
}

\section{Introduction}

Encephalitis and/or meningitis are central nervous system (CNS) diseases that involve inflammation of the brain parenchyma and/or meninges (Thompson et al., 2012). Viruses that cross the blood-brain barrier (BBB) cause the viral infections of CNS, which is the most prevalent cause of encephalitis and/or meningitis in pediatrics, called viral encephalitis and/or viral meningitis (VEVM) (Thompson et al., 2012; Kong et al., 2015; Ai et al., 2017).

The clinical diagnosis of VEVM is established by clinical manifestation, laboratory detection, clinical examination, and neuroimaging (Mutton and Guiver, 2011). The etiological diagnosis of VEVM is mainly based on the identification of viruses or viral antibodies in cerebrospinal fluid (CSF) obtained by lumbar puncture (LP); however, up to $60 \%$ of cases were etiologically unexplained (Thompson et al., 2012; Lyons, 2018; Wright et al., 2019). Common pediatric diseases, including fever, headache, epilepsy, bacterial meningitis, febrile convulsion, etc., have similar clinical features and unknown etiologies as VEVM, resulting in the difficulty of the discrimination between these conditions and VEVM (Mutton and Guiver, 2011; Thompson et al., 2012; Venter et al., 2014; Ye et al., 2016; Kennedy et al., 2017). In addition, the injuries caused by needles and other possible complications, including postdural puncture headache, back pain, etc., lead to a lower acceptance of LP, especially in children (Engelborghs et al., 2017).

Over 700 taxa comprise the oral microbiota, which is a complex ecosystem and has important influences on human health and physiology (Sampaio-Maia et al., 2016). The traits of oral microbiota, consisting of alpha/beta diversity, composition, relative abundance, and functional prediction, have been confirmed to be associated with various diseases and to be helpful in their diagnosis, including viral infectious diseases like coronavirus disease 2019 (Docktor et al., 2012; Qiao et al., 2018; Lee et al., 2020; Zhang et al., 2020; Ma et al., 2021). Viruses have been proved to be able to alter microbial adherence, colonization, and host immune responses (Allen et al., 2014). Besides, oral microbial dysbiosis also has potential impacts on the integrity of $\mathrm{BBB}$ and the CNS, suggesting that oral microbiota might play a significant role in the mechanisms of neurological diseases (Shoemark and Allen, 2015; Qiao et al., 2018).

The relationship between VEVM and oral microbiota remains unclear. The current study aimed to explore the alterations of oral microbiota and their diagnostic performance in Chinese children with VEVM. 


\section{Materials and Methods}

\section{Study design and participants}

The hospital-based study recruited children who were given LPs in the Children's Hospital of Fudan University in Shanghai, China, from January 2018 to December 2019. The study protocol was reviewed and approved by the ethical committee of the School of Public Health, Fudan University (IRB\#2017-09-0637).

Children included in this study were: (1) aged from one month to 16 years old (the upper age limit of the children allowed to visit the hospital), (2) both CSF and oral swab samples can be collected, and (3) informed consents from the participants' guardians were obtained. Children were excluded from this study when they received antibiotic treatments within one week before having LPs. A total of 512 children were recruited, 398 of whom met the inclusion/exclusion criteria above.

Oral swab samples were collected from the buccal mucosa using sterile swabs immediately after the LPs. The swabs were labeled with serial numbers and immediately frozen at $-20^{\circ} \mathrm{C}$, then transported to the laboratory and stored at $-80^{\circ} \mathrm{C}$ within $24 \mathrm{~h}$. General sociodemographic and clinical data were collected from the hospital's electronic medical records.

\section{Clinical and etiological diagnosis of VEVM}

Clinical diagnosis for viral encephalitis was established based on the diagnostic criteria (Wang, 2013; Jiang et al., 2015), including (1) acute onset, (2) symptoms of cerebral parenchyma damage including fever, headache, projectile vomiting, lethargy, convulsion, conscious disturbance, and/or focal neurological signs, (3) focal or diffuse abnormalities in electroencephalogram (EEG), (4) brain edema or focal/diffuse lesions in computed tomography (CT) and/or magnetic resonance imaging (MRI) tests, (5) elevated CSF leukocyte and protein levels, and (6) the lack of evidence for bacterial, tubercle bacillus, or fungal infection. And the diagnostic criteria of viral meningitis were based on the followings (Tuppeny, 2013; Ai et al., 2017): (1) acute onset, (2) symptoms such as fever, headache, vomiting, and/or nuchal rigidity without parenchymal involvement, (3) elevated CSF leukocyte and elevated or normal CSF protein levels, and (4) the lack of evidence for bacterial, tubercle bacillus, or fungal infection. Among the 398 eligible children, 68 children were clinically diagnosed with VEVM (denoted as VEVMc), and 330 children were diagnosed with other diseases, including fever, headache, epilepsy, febrile convulsion, etc. For all VEVMc children, we applied polymerase chain reaction (PCR) or reverse transcription polymerase chain reaction (RT-PCR) to detect viruses in CSF, and the detailed experimental methods have been reported in our previous study (Zhu et al., 2021). The measured viruses, including enterovirus (EV), herpes simplex virus (HSV)-1, HSV-2, mumps virus (MuV), Epsteinbarr virus, cytomegalovirus, and varicella-zoster virus, which were the most prevalent pathogens among children with VEVM in China (Zhang et al., 2013; Kong et al., 2015; Xie et al., 2015; Ai et al., 2017). Among the 68 children with VEVMc, 43 children were positive in PCR or RT-PCR results (denoted as VEVMP), and 25 children were negative in PCR or RT-PCR results (denoted as VEVMN). From children with other diseases $(n=330)$, a frequency-matched method was used to match the VEVMc group on age, gender, and diagnostic season with a ratio of $1: 1$, which produced the control group $(n=68)$. The study flowchart is shown in Fig. 1.

\section{DNA extraction and 16S rRNA gene sequencing}

For the 136 oral swab samples ( 68 children with VEVMc and 68 controls), genomic DNA was extracted using the cetyltrimethylammonium bromide (CTAB) method. The V3-V4 hypervariable regions of $16 \mathrm{~S}$ rRNA genes were then amplified using the primers (341F-CTAYGGGRBGCASCAG and 806R-GGACTACNNGGGTATCTAAT) with specific barcodes, followed by sequencing on an Ion $\mathrm{S} 5^{\mathrm{TM}}$ XL platform (Thermofisher Scientific Co. Ltd.) to generate 600 bp singleend reads. The reads were assigned to their corresponding samples according to their unique barcodes, and then the barcodes and primer sequences were removed.

\section{Statistical and bioinformatics analyses}

The comparisons of the sociodemographic and clinical characteristics were performed between the VEVMc group and

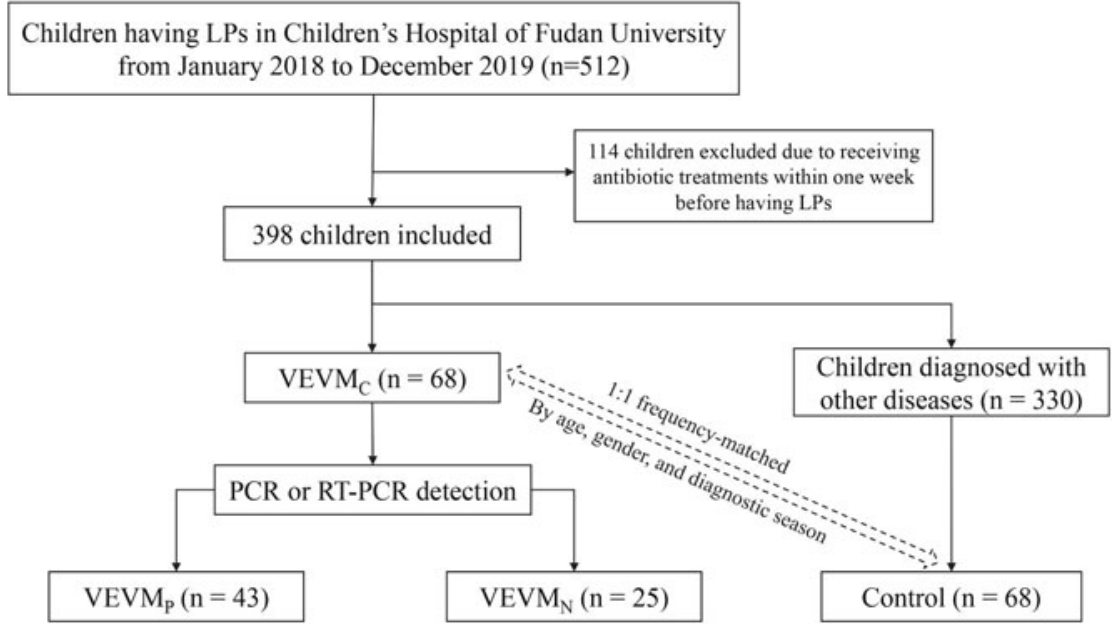

Fig. 1. Flowchart of study participants. The flowchart shows the enrollment of children for subsequent oral microbiota analysis. LP, lumbar puncture; PCR, polymerase chain reaction; RT-PCR, reverse transcription polymerase chain reaction. 
the control group, as well as among the VEVMP group, the VEVMN group, and the control group. Continuous variables with a normal distribution were compared by Student's t test for two groups or one-way ANOVA (Analysis of Variance) for three groups. Continuous variables with a non-normal distribution were compared by the Wilcoxon test for two groups or the Kruskal-Wallis test for three groups. Normality was assessed by the Shapiro-Wilk test. The proportions of categorical variables were compared by the Chi-square test (or Fisher's exact test). When the comparisons among all three groups showed significant differences, the pairwise multiple comparisons were performed by the post-hoc test with the false discovery rate (FDR) correction.

The sequence data were processed using the Quantitative Insights into Microbial Ecology 2 (QIIME 2, Version 2019.7) platform, with plugins DADA2, feature-table, feature-classifier, and phylogeny (Bolyen et al., 2019). The quality control and the construction of the amplicon sequence variant (ASV) table were conducted using the DADA2 pipeline (Callahan et al., 2016). After filtering sequences with low abundances (less than ten reads), sequences only present in a few samples (less than five samples), and chimeras, taxonomic classification was assigned to ASVs using the Silva database (version 132).

Further analyses and visualizations were performed using $R$ 3.6.1. Alpha diversity analyses, including the calculation of Shannon index, observed species, Evenness, and Faith phylogenetic diversity (PD), were conducted to assess the richness and evenness of oral microbiota. Statistical differences were determined with the Wilcoxon test for two groups or the Kruskal-Wallis test for three groups. Principal coordinates analyses (PCoA) were conducted to examine beta diversity metrics across groups based on the weighted UniFrac distance, unweighted UniFrac distance, Bray-curtis dissimilarity, and Jaccard dissimilarity metrics. PERMANOVA (Permutational Multivariate ANOVA) tests were used to evaluate the significance of beta diversity differences among groups. When the comparisons among all three groups showed significant differences, the $P$ values from pairwise PERMANOVA tests were corrected for multiple testing using FDR.

Compositional analyses with stacked bar charts were conducted to exhibit taxonomic compositions of the oral microbiota in different groups at the phylum, family, genus, and species levels. Linear discriminant analysis (LDA) with effect size (LEfSe) tests were performed to identify the differentially abundant taxa between groups, using LDA scores $>2.0$ as a threshold of significance (Segata et al., 2011). Cladograms of a taxonomy-based tree were used to visualize the differentially abundant taxa extending from the phylum to genus levels, and bar charts were used to demonstrate these taxa at the genus and species level.

PICRUSt2 (Phylogenetic Investigation of Communities by Reconstruction of Unobserved States 2) (Douglas et al., 2020) was applied to predict the functions of oral microbiota using Kyoto Encyclopedia of Genes and Genomes (KEGG) orthologs. The predicted pathways were grouped by three hierarchical levels, and the differential pathways were found using Welch's t-test with FDR. Spearman correlation analysis was performed to assess the relationships between discriminative genera and pathways with FDR correction.
In order to examine whether VEVM can be classified based on the relative abundances of oral microbiota at the genus level, the random forest (RF) machine learning algorithm was used to construct a model with ten-fold cross-validation (five replicates). Two-thirds of the samples were selected to train the classifier by sampling with replacement, and the remaining samples were used for validation. A mean decrease accuracy (MDA) value of each variable was calculated and ranked in a descending order to investigate the degree of its contribution to the RF model. The minimum number of discriminative taxa used for the model was dependent on the minimum classification error rate. The receiver operating characteristic (ROC) curve was then constructed and the area under the ROC curve (AUC) was calculated to evaluate the RF classifier (Robin et al., 2011). The statistical significance of the improvement in AUC was determined using Delong's test (DeLong et al., 1988). All tests were two-tailed, and the $P$ values (or FDR $q$ values) $<0.05$ were considered statistically significant.

\section{Results}

\section{Participants' characteristics}

The profiles of demographic and clinical characteristics of the participants were presented in Supplementary data Table S1. Among the participants, the headache was more often observed in the VEVMc and VEVMP group than in the control group $(P<0.05$, Supplementary data Table $S 1$ and $q<$ 0.05 , Supplementary data Fig. S1), while cough and convulsion were more frequent in the control group than in the VEVMc and VEVMP group $(P<0.05$, Supplementary data Table S1 and $q<0.05$, Supplementary data Fig. S1). Higher CSF leukocyte count, CSF protein level, and $\gamma$-glutamyl transpeptidase were found in the VEVMc and VEVMp group $(P<$ 0.05 , Supplementary data Table $S 1$ and $q<0.05$, Supplementary data Fig. S2). Compared to the control group, the blood platelet count was higher only in the VEVMc group $(P<0.05)$, while the proportion of abnormal C-reactive protein was lower only in the VEVMc group $(P<0.05)$ (Supplementary data Table S1). The frequency of vomiting was higher only in the VEVMp group than in the control group $(q<0.05$, Supplementary data Fig. S2). Other clinical characteristics were similar between the VEVMc/VEVMr group and the control group. Moreover, there were significant differences in headache, vomiting, and CSF leukocyte count between the VEVMP group and the VEVMN group $(q<0.05$, Supplementary data Figs. S1-S2); and it was also significantly different in CSF leukocyte count, CSF protein level, and blood platelet count between the VEVMN group and the control group $(q<0.05$, Supplementary data Fig. S2).

\section{Alpha and beta diversities of oral microbiota}

Among the 136 participants, a total of 5,643,390 raw sequence reads were obtained, and the quality filtering retained $5,213,070$ reads with an average of 38,331 reads per sample (ranges: 18,097 reads to 52,787 reads). The Shannon index, observed species, Evenness, and Faith PD of oral microbiota were similar between the VEVMc and the control group $(P>$ 

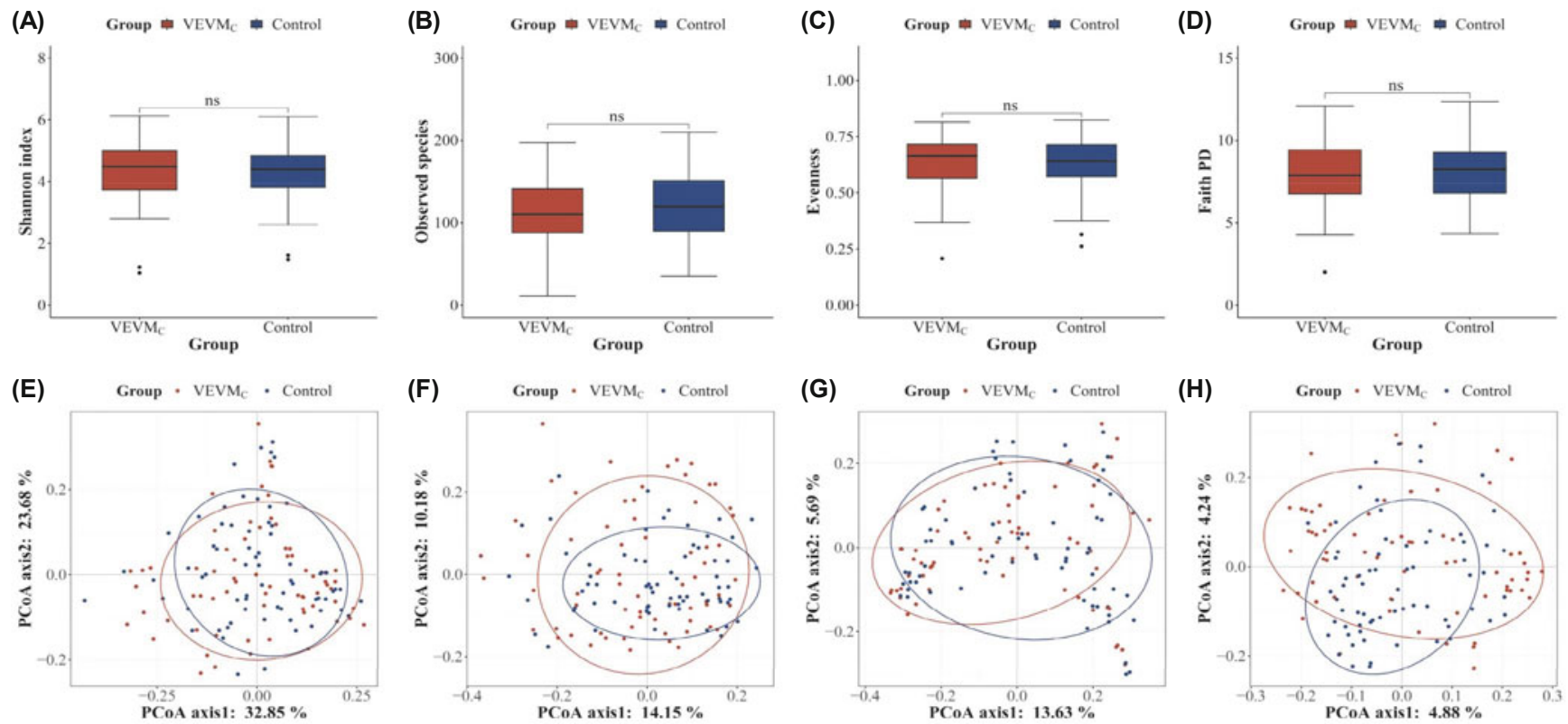

Fig. 2. Comparisons of alpha and beta diversities of the oral microbiota between the VEVMc group and the control group. Boxplots display the comparisons of alpha diversity metrics of (A) Shannon index, (B) observed species, (C) Evenness, and (D) Faith PD between two groups. Boxes show the interquartile ranges, lines inside the boxes show medians, and circles show outliers. PCoA was used to visualize compositional relationships between two groups according to beta diversity metrics of $(\mathrm{E})$ weighted UniFrac distance $\left(P=0.734, \mathrm{R}^{2}=0.004\right),(\mathrm{F})$ unweighted UniFrac distance $\left(P<0.001, \mathrm{R}^{2}=0.025\right)$, $(\mathrm{G}) \mathrm{Bray}-\mathrm{curtis}$ dissimilarity $\left(P=0.045, \mathrm{R}^{2}=0.011\right)$, and $(\mathrm{H})$ Jaccard dissimilarity $\left(P<0.001, \mathrm{R}^{2}=0.017\right)$. The percentage on axis 1 and 2 labels are the proportion of variance explained by that axis. Faith PD, Faith phylogenetic diversity; ns, no significance $(P>0.05)$; PCoA, principal coordinates analysis

0.05, Fig. 2A-D). Similar results were observed among the VEVMp group, the VEVMN group, and the control group $(P>0.05$, Supplementary data Fig. S3A-D).

The differences of the beta diversity metrics, including unweighted UniFrac distance $\left(P<0.001, \mathrm{R}^{2}=0.025\right)$, Braycurtis dissimilarity $\left(P=0.045, \mathrm{R}^{2}=0.011\right)$, and Jaccard dissimilarity $\left(P<0.001, \mathrm{R}^{2}=0.017\right)$, were significant between the VEVMc group and the control group (Fig. 2E-H). Similar results were also obtained among the VEVMp group, the VEVMN group, and the control group (unweighted UniFrac distance: $P<0.001, \mathrm{R}^{2}=0.035$; Bray-curtis dissimilarity: $P=$ $0.013, \mathrm{R}^{2}=0.022$; Jaccard dissimilarity: $P<0.001, \mathrm{R}^{2}=0.027$, Supplementary data Fig. S3E-H), as shown in the PCoA plots. Further multiple pairwise comparisons showed that there were significant differences in the beta diversity metrics based on Bray-curtis dissimilarity and Jaccard dissimilarity between any two groups $(q<0.05)$, while the metric of unweighted UniFrac distance was only significantly different in the VEVMP and VEVMN group compared with the control group $(q<$ 0.05) (Supplementary data Table S2).

\section{Taxa of oral microbiota}

Among the 136 participants, 10 phyla, 18 classes, 35 orders, 65 families, 147 genera, and 235 species were obtained based on the sequences. At the phylum level, the proportions of the top five phyla (Firmicutes, Proteobacteria, Bacteroidetes, Actinobacteria, and Fusobacteria) in total were 98.6\%, 99.1\%, $97.8 \%$, and $98.5 \%$ respectively for the VEVMc group, the VEVMP group, the VEVMN group, and the control group
(A)

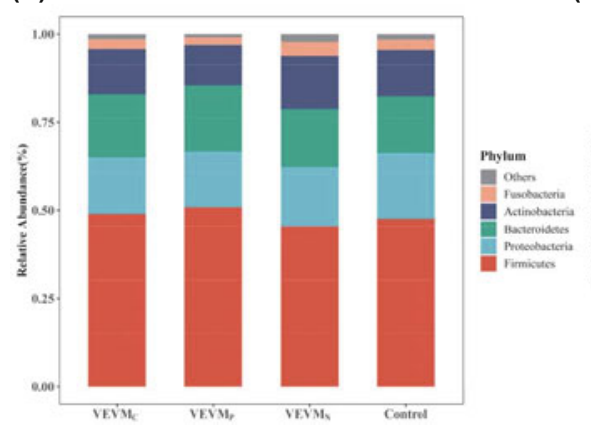

(B)

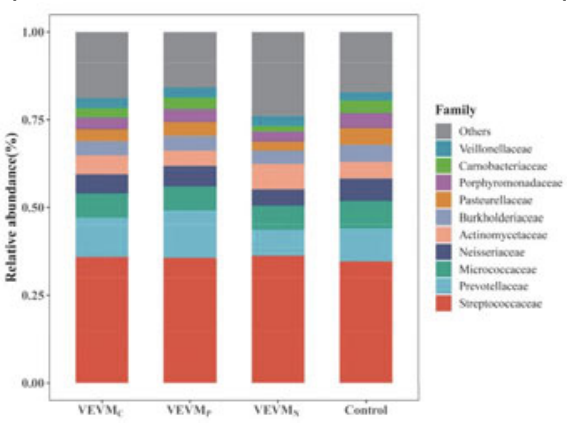

(C)

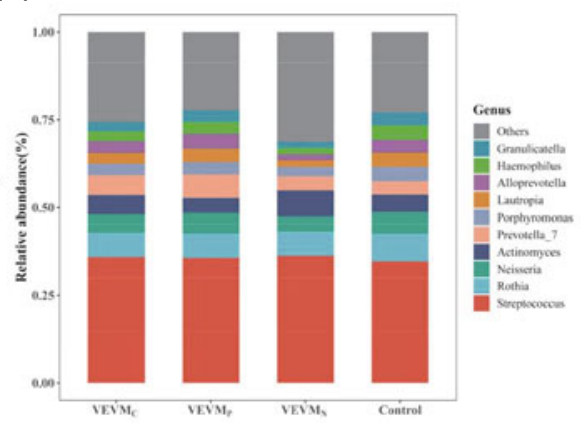

Fig. 3. Compositions of the oral microbiota. Average relative abundances of (A) the top five most abundant phyla, (B) the top 10 most abundant families, and (C) the top 10 most abundant genera in the VEVMc group, the VEVMp group, the VEVMN group, and the control group. 
(A)

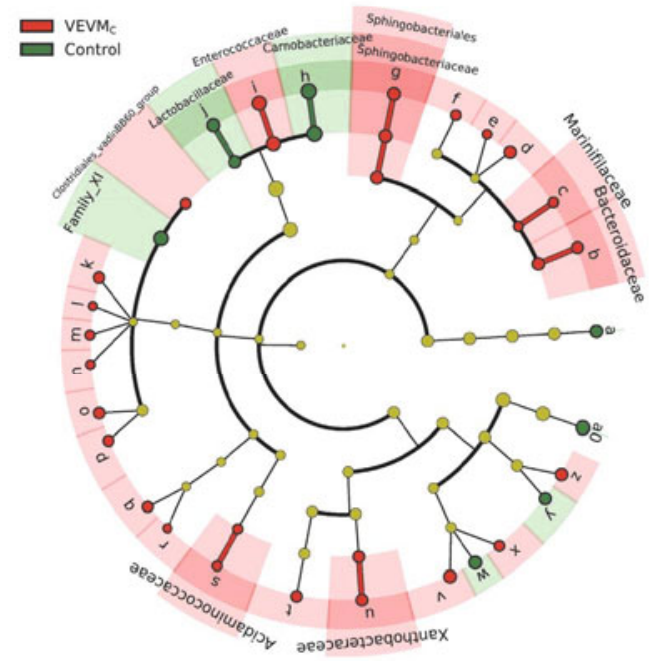

(B)

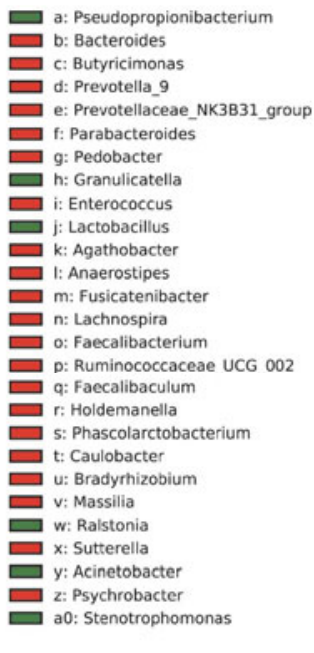

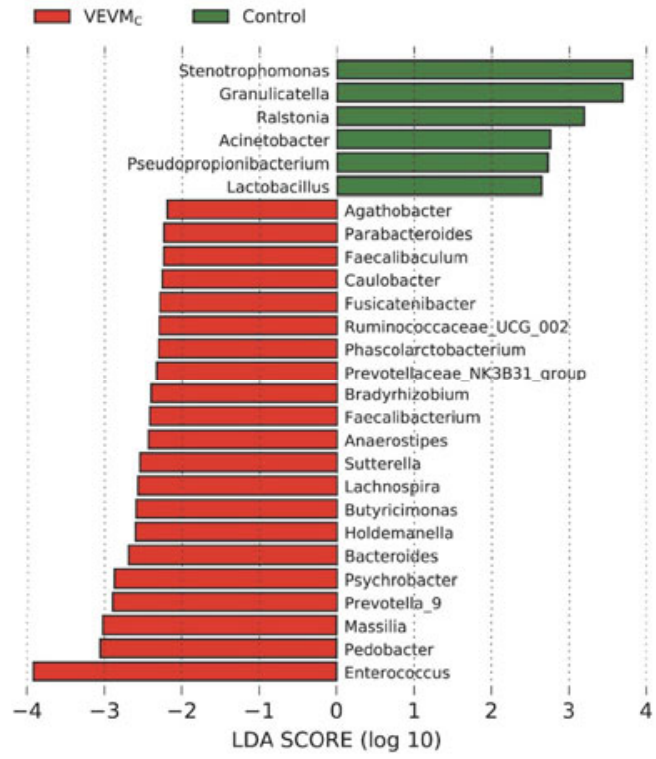

Fig. 4. LEfSe analysis results of oral microbiota between the VEVMc group and the control group. (A) Taxonomic cladogram of the differentially abundant taxa from the phylum to genus levels. From the interior to the exterior, each layer represents the phylum, class, order, family, and genus level. (B) Bar chart of differentially abundant taxa at the genus level. LEfSe, linear discriminant analysis with effect size; LDA, linear discriminant analysis.

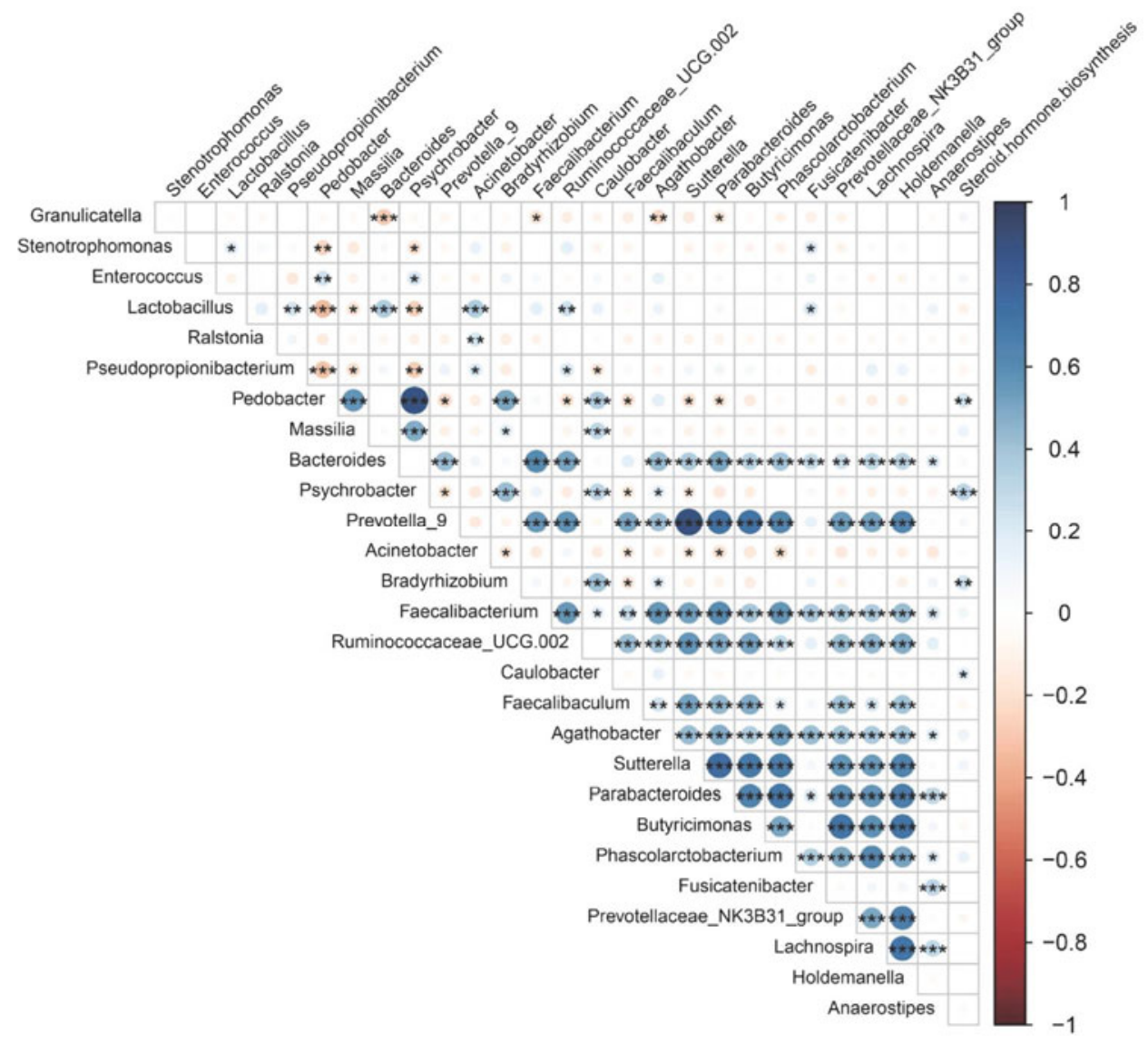

Fig. 5. Correlation between steroid hormone biosynthesis and differentially abundant genera between the VEVMc group and the control group. The relative abundances of differentially abundant genera between the VEVMc group and the control group were analyzed for covariation with steroid hormone biosynthesis using Spearman correlation analysis. The correlation coefficient is indicated by a color gradient from red (negative correlation) to blue (positive correlation). ${ }^{*} P<0.05 ;{ }^{* *} P<0.01 ;{ }^{* *} P<0.001$. 
(Fig. 3A). At the family level, the proportions of the top 10 families (Streptococcaceae, Prevotellaceae, Micrococcaceae, Neisseriaceae, Actinomycetaceae, Burkholderiaceae, Pasteurellaceae, Porphyromonadaceae, Carnobacteriaceae, and Veillonellaceae) in total were $81.1 \%, 84.1 \%, 76.1 \%$, and $82.8 \%$ for the $\mathrm{VEVM}_{\mathrm{C}}$ group, the VEVMp group, the VEVMN group, and the control group, respectively (Fig. 3B). At the genus level, the proportions of the top 10 genera (Streptococcus, Rothia, Neisseria, Actinomyces, Prevotella_7, Porphyromonas, Lautropia, Alloprevotella, Haemophilus, and Granulicatella) in total were $74.4 \%, 77.7 \%, 68.7 \%$, and $77.0 \%$ for the VEVMc group, the VEVMP group, the VEVMN group, and the control group, respectively (Fig. 3C).

Further, 60 differentially taxa that varied significantly in abundance between the VEVMc group and the control group based on LEfSe analysis (LDA scores > 2.0) were identified. These taxa included one order, 10 families, 27 genera, and 22 species (Fig. 4A). 27 differentially abundant taxa at the genus level were shown by bar charts (Fig. 4B), almost completely covering the 20 differentially abundant genera between the VEVMr group and the control group (except Corynebac- terium, Supplementary data Fig. S4B). The result of LEfSe analysis between the VEVMN group and the control group was shown in Supplementary data Fig. S5, which was slightly different from the result obtained between the VEVMc group and the control group. The composition and the differentially abundant taxa at the species level among groups were also shown in Supplementary data Fig. S6.

\section{Functional prediction of oral microbiota}

The functional prediction results showed that the pathway of steroid hormone biosynthesis exhibited higher abundances in both the VEVMc group $(q=0.020)$ and the VEVMP group $(q=0.003)$ compared to the control group. VEVMc-enriched genera, including Psychrobacter $(R=0.312, q<0.001)$, Bradyrhizobium $(R=0.247, q=0.004)$, Pedobacter $(R=0.229, q=$ $0.007)$, and Caulobacter $(R=0.169, q=0.049)$, were positively correlated to the pathway abundance of steroid hormone biosynthesis (Fig. 5). No pathways differed significantly between the VEVMN group and the control group in abundance $(q>0.05)$.
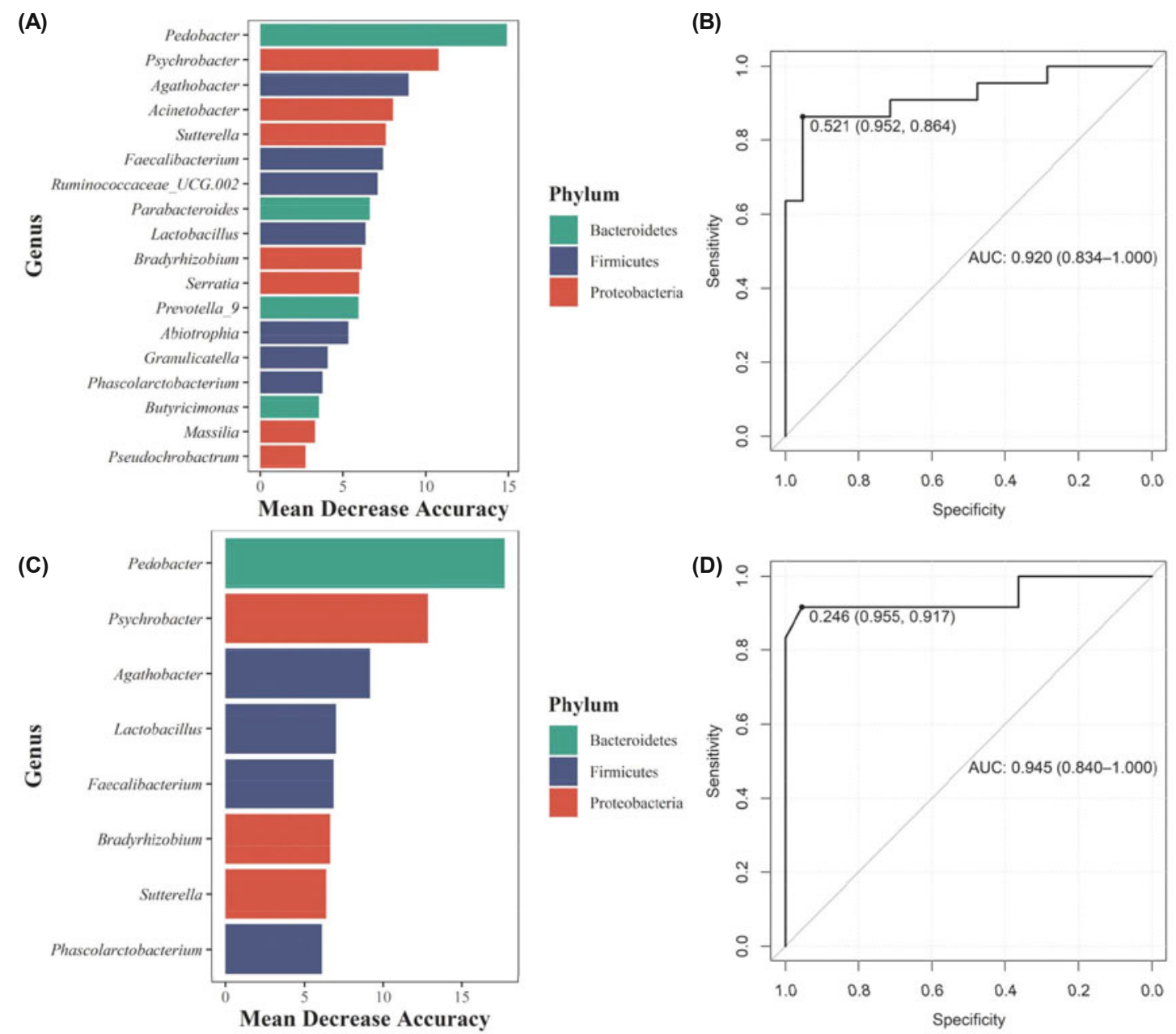

Fig. 6. Discriminative genera identified by RF model and ROC curve. The genera ranking in descending order of mean decrease accuracy based on their relative abundances to classify (A) the $\mathrm{VEVM}_{\mathrm{C}}$ group and the control group and (C) the VEVMP group and the control group. The ROC curve of the RF model between (B) the VEVM $\mathrm{C}$ group and the control group and (D) the VEVMP group and the control group. RF, random forest; ROC, receiver operating characteristic. 


\section{Oral microbiota-based predictive model for VEVM}

The relative abundances of eighteen discriminative genera were used to establish the RF model for predicting VEVMc (Fig. 6A). Most of these genera were also discovered by the analysis of LEfSe except Serratia, Abiotrophia, and Pseudochrobactrum (Fig. 4B). The AUC of the RF model was 0.920 (95\% confidence interval [CI]: 0.834-1.000). At the optimal cutoff value of 0.521 , the model above demonstrated a $90.7 \%$ predictive accuracy for discriminating children with VEVMc from controls, with a sensitivity of $86.4 \%$ and a specificity of 95.2\% (Fig. 6B). The positive predictive value (PPV) and negative predictive value (NPV) were $95.0 \%$ and $87.0 \%$, respectively.

Eight discriminative genera, appearing also in the results of LEfSe (Supplementary data Fig. S4B), were used in constructing the RF model to distinguish children with VEVMP from controls with the same method (Fig. 6C). The AUC of the RF model in predicting VEVMP was 0.945 (95\% CI: 0.8401.000 ), with a sensitivity of $91.7 \%$ and a specificity of $95.5 \%$ (Fig. 6D). The AUC for predicting VEVM has been improved from 0.920 to 0.945 , but without statistical significance (Delong's test, $P=0.533$ ).

\section{Discussion}

The current study identified different beta diversity, differentially abundant taxa, and a higher pathway abundance of the oral microbiota in children with VEVM compared to controls; the microbiota-based model showed a relatively good performance in predicting either clinically diagnosed VEVM or its subset with definite etiologies.

In this study, children with clinically diagnosed VEVM exhibited different beta diversity compared with controls (Fig. 2 ), which were manifested by the differentially abundant taxa, including Enterococcus, Pedobacter, Massilia, Prevotella_9, Psychrobacter, Butyricimonas, Bradyrhizobium (Fig. 4), etc. Children with clinically diagnosed VEVM also showed a higher pathway abundance of the steroid hormone biosynthesis predicted from the oral microbiota $(q=0.020)$. Similar results were also obtained between children with etiologically diagnosed VEVM and controls (Supplementary data Figs. S3 and S4). The microbiota-based models predicted for clinically diagnosed VEVM with an AUC of 0.920 (95\% CI: 0.834-1.000) and etiologically diagnosed VEVM with an AUC of 0.945 (95\% CI: 0.840-1.000) (Fig. 6).

Oral microbiota in children altered with increasing age (Dzidic et al., 2018); however, the compositions have developed into adult-like patterns among children older than one month (Chu et al., 2017). In our design, children older than one month were included, and the age has been matched between the VEVMc group and the control group. The difference in the beta diversity of oral microbiota between the VEVM group and the control group indicated their different oral microbial compositions in our study. Similar results have been found in other viral infections, like coronavirus (Ma et al., 2021), hepatitis B virus (Ling et al., 2015), and HSV-1 (Lee et al., 2020). However, the decreased alpha diversity of the oropharyngeal microbiota was found in patients with coronavirus disease 2019 (Ma et al., 2021), which was not observed in our results, suggesting that the alterations of oral microbiota in children with viral infections may be complex or virusspecific. Several VEVM-enriched microbial taxa found in the current research have been reported in relation to specific human diseases previously. For example, Enterococcus in CSF could be one of the causes for bacterial encephalitis, although it was relatively uncommon (Granerod et al., 2010); Bradyrhizobium was present in the colon-biopsy specimens of patients with cord colitis but not in controls, and was considered as an opportunistic human pathogen (Bhatt et al., 2013).

Viral infections may cause inflammation and lead to pronounced neuro-endocrine and metabolic alterations (Joosten et al., 2000). Mehta et al. (2015) reported the CSF cortisol (a steroid stress-response hormone) level in patients with viral meningitis was higher than that in controls. In addition, oral microbiota has been proved to synthesize enzymes needed for steroid hormone synthesis and catabolism (Markou et al., 2009). The current study found the pathway of steroid hormone biosynthesis predicted by the oral microbiota was enriched in children with VEVM, and positively correlated with some VEVM-enriched genera. This finding suggests the potential mechanism between steroid hormone biosynthesis and VEVM pathogenesis.

Low viral contents in CSF, difficulties in viral culture, and initial non-specific clinical features of VEVM can result in the misdiagnosis and the delayed treatment of VEVM (Kong et al., 2015; Gao et al., 2017). In our study, children with VEVM presented with many similar clinical characteristics when compared to those with other diseases, and the similarities also appeared between children with VEVMP and children with VEVMN (Supplementary data Table S1 and Figs. S1-S2). These similarities implied the difficulty in differentiating VEVM from other common pediatric disorders and making a clear distinction between VEVMP and VEVMN in routine clinical practices. Thus, new methods for the diagnosis of VEVM are still required. Xing et al. (2020) used nextgeneration metagenomic sequencing to diagnose viral encephalitis, achieving an AUC of 0.659 (95\% CI: 0.566-0.751). Mori et al. (2017) found serum vascular endothelial growth factor was able to discriminate virus-positive and virus-negative encephalitis in a study of Bangladeshi children with an AUC of 0.82 (95\% CI: 0.66-0.98). The primary infection and replication of the major causative agents of VEVM usually occur in the oral cavity (Thompson et al., 2012; Rozenberg, 2013). Hence, the alterations of oral microbiota might appear preceding the onset of clinical symptoms or the changes of clinical indicators in CSF (Thompson et al., 2012). However, the utility of oral microbiota in the diagnosis of VEVM was rarely reported in previous researches. The current study established a predictive method for distinguishing children with VEVM from those with other similar diseases based on the oral microbial traits with a higher value of AUC, which implied that our method may be superior to those reported previously.

Our findings have important implications for current clinical practices. Firstly, the oral microbiota-based predictive method may help clinicians in the preliminary diagnosis and initiation of antiviral treatments earlier for VEVM, which probably brings out a better prognosis and reduces the unnecessary injuries or complications induced by invasive LPs. 
Secondly, the oral microbiota-based method performed well in predicting the VEVM, which implied that the studies on the methods of identifying viruses from the oral cavity are encouraging. Additionally, oral microbial metabolism might be involved in the pathogenesis of VEVM, and the exact mechanisms are worthy of further exploration in the future.

This study nevertheless exhibited certain limitations. Firstly, there was a possible patient selection bias in this study. Children who refused to take LPs or donate oral samples and who received antibiotic treatments within one week before having LPs were excluded. Secondly, the study only sampled a single site in the oral cavity. Oral microbiota exhibits different compositions at different locations including in the saliva, dental plaque, subgingival sulcus, and mucosa (Santigli et al., 2017). Samplings at different oral cavity niches may offer more complete microbiota information. Thirdly, due to the low incidence of VEVM and a broad age range of participants, future studies with larger sample sizes may finely discover the role of oral microbiota in children with VEVM of different ages. Moreover, the oral microbiota was also affected by food intake (Katagiri et al., 2019), but this information was not available in our study. Comprehensive food investigations are needed to clarify the exact role of food intake in oral microbiota in future studies.

In conclusion, the current study found VEVM-enriched oral microbiota and established a relatively effective model in predicting VEVM in Chinese children, which will provide new insights into the role of oral microbiota in VEVM.

\section{Acknowledgements}

This work was supported by the National Key Research and Development Program of China [grant numbers 2021YFC2701800, 2017YFC1200203] and the National Natural Science Foundation of China [grant numbers 82173582, 81773490, 81373065].

\section{Conflict of Interest}

The authors declare no conflict of interests.

\section{Ethical Statements}

The study was approved by the ethical committee of the School of Public Health at Fudan University (IRB\#2017-090637). Informed consents from participants' guardians were obtained.

\section{References}

Ai, J., Xie, Z., Liu, G., Chen, Z., Yang, Y., Li, Y., Chen, J., Zheng, G., and Shen, K. 2017. Etiology and prognosis of acute viral encephalitis and meningitis in Chinese children: a multicentre prospective study. BMC Infect. Dis. 17, 494.

Allen, E.K., Koeppel, A.F., Hendley, J.O., Turner, S.D., Winther, B., and Sale, M.M. 2014. Characterization of the nasopharyngeal microbiota in health and during rhinovirus challenge. Microbiome
2,22 .

Bhatt, A.S., Freeman, S.S., Herrera, A.F., Pedamallu, C.S., Gevers, D., Duke, F., Jung, J., Michaud, M., Walker, B.J., Young, S., et al. 2013. Sequence-based discovery of Bradyrhizobium enterica in cord colitis syndrome. N. Engl. J. Med. 369, 517-528.

Bolyen, E., Rideout, J.R., Dillon, M.R., Bokulich, N.A., Abnet, C.C., Al-Ghalith, G.A., Alexander, H., Alm, E.J., Arumugam, M., Asnicar, F., et al. 2019. Reproducible, interactive, scalable and extensible microbiome data science using QIIME 2. Nat. Biotechnol. 37, 852-857.

Callahan, B.J., McMurdie, P.J., Rosen, M.J., Han, A.W., Johnson, A.J., and Holmes, S.P. 2016. DADA2: High-resolution sample inference from Illumina amplicon data. Nat. Methods 13, 581-583.

Chu, D.M., Ma, J., Prince, A.L., Antony, K.M., Seferovic, M.D., and Aagaard, K.M. 2017. Maturation of the infant microbiome community structure and function across multiple body sites and in relation to mode of delivery. Nat. Med. 23, 314-326.

DeLong, E.R., DeLong, D.M., and Clarke-Pearson, D.L. 1988. Comparing the areas under two or more correlated receiver operating characteristic curves: a nonparametric approach. Biometrics 44, 837-845.

Docktor, M.J., Paster, B.J., Abramowicz, S., Ingram, J., Wang, Y.E., Correll, M., Jiang, H., Cotton, S.L., Kokaras, A.S., and Bousvaros, A. 2012. Alterations in diversity of the oral microbiome in pediatric inflammatory bowel disease. Inflamm. Bowel Dis. 18, 935-942.

Douglas, G.M., Maffei, V.J., Zaneveld, J.R., Yurgel, S.N., Brown, J.R., Taylor, C.M., Huttenhower, C., and Langille, M.G.I. 2020. PICRUSt2 for prediction of metagenome functions. Nat. Biotechnol. 38, 685-688.

Dzidic, M., Collado, M.C., Abrahamsson, T., Artacho, A., Stensson, M., Jenmalm, M.C., and Mira, A. 2018. Oral microbiome development during childhood: an ecological succession influenced by postnatal factors and associated with tooth decay. ISME J. 12, 2292-2306.

Engelborghs, S., Niemantsverdriet, E., Struyfs, H., Blennow, K., Brouns, R, Comabella, M., Dujmovic, I., van der Flier, W., Frölich, L., Galimberti, D., et al. 2017. Consensus guidelines for lumbar puncture in patients with neurological diseases. Alzheimers Dement. 8, 111-126.

Gao, Q.L., Ma, Y.X., Yuan, D.W., Zhang, Q.C., Zeng, J., and Li, H. 2017. MicroRNA-125b in peripheral blood: a potential biomarker for severity and prognosis of children with viral encephalitis. Neurol. Sci. 38, 1437-1444.

Granerod, J., Ambrose, H.E., Davies, N.W., Clewley, J.P., Walsh, A.L., Morgan, D., Cunningham, R., Zuckerman, M., Mutton, K.J., Solomon, T., et al. 2010. Causes of encephalitis and differences in their clinical presentations in England: a multicentre, populationbased prospective study. Lancet Infect. Dis. 10, 835-844.

Jiang, Z.F., Shen, K.L., and Shen, Y. 2015. Zhu Futang Practice of Pediatrics, 8th edn. People's Medical Publishing House, Beijing, China.

Joosten, K.F., de Kleijn, E.D., Westerterp, M., de Hoog, M., Eijck, F.C., Hop, W.C.J., Voort, E.V., Hazelzet, J.A., and HokkenKoelega, A.C. 2000. Endocrine and metabolic responses in children with meningoccocal sepsis: striking differences between survivors and nonsurvivors. J. Clin. Endocrinol. Metab. 85, 37463753.

Katagiri, S., Shiba, T., Tohara, H., Yamaguchi, K., Hara, K., Nakagawa, K., Komatsu, K., Watanabe, K., Ohsugi, Y., Maekawa, S., et al. 2019. Re-initiation of oral food intake following enteral nutrition alters oral and gut microbiota communities. Front. Cell. Infect. Microbiol. 9, 434.

Kennedy, P.G.E., Quan, P.L., and Lipkin, W.I. 2017. Viral encephalitis of unknown cause: current perspective and recent advances. Viruses 9, 138.

Kong, X., Zhang, L., Liu, K., Chen, H., Li, B., Wu, R., and Ji, C. 2015. Epidemiological features of viral encephalitis in Cangzhou of 
China with use of multiplex RT-PCR for five RNA viruses. $J$. Virol. Methods 222, 178-181.

Lee, A., Hong, J., Shin, D.Y., Koh, Y., Yoon, S.S., Kim, P.J., Kim, H.G., Kim, I., Park, H.K., and Choi, Y. 2020. Association of HSV-1 and reduced oral bacteriota diversity with chemotherapy-induced oral mucositis in patients undergoing autologous hematopoietic stem cell transplantation. J. Clin. Med. 9, 1090.

Ling, Z., Liu, X., Cheng, Y., Jiang, X., Jiang, H., Wang, Y., and Li, L. 2015. Decreased diversity of the oral microbiota of patients with hepatitis B virus-induced chronic liver disease: a pilot project. Sci. Rep. 5, 17098.

Lyons, J.L. 2018. Viral Meningitis and Encephalitis. Continuum (Minneap Minn) 24, 1284-1297.

Ma, S., Zhang, F., Zhou, F., Li, H., Ge, W., Gan, R., Nie, H., Li, B., Wang, Y., Wu, M., et al. 2021. Metagenomic analysis reveals oropharyngeal microbiota alterations in patients with COVID-19. Sig. Transduct. Target. Ther. 6, 191.

Markou, E., Eleana, B., Lazaros, T., and Antonios, K. 2009. The influence of sex steroid hormones on gingiva of women. Open Dent. J. 3, 114-119.

Mehta, A., Mahale, R.R., Sudhir, U., Javali, M., and Srinivasa, R. 2015. Utility of cerebrospinal fluid cortisol level in acute bacterial meningitis. Ann. Indian Acad. Neurol. 18, 210-214.

Mori, D., Khanam, W., Sheikh, R.A., Tabib, S., Ikebe, E., Hossain, M.M., Iha, H., and Ahmed, K. 2017. Increased serum vascular endothelial growth factor is associated with acute viral encephalitis in Bangladeshi children. Sci. Rep. 7, 16181.

Mutton, K. and Guiver, M. 2011. Laboratory techniques for human viral encephalitis diagnosis. Infect. Disord. Drug Targets 11, $206-$ 234.

Qiao, Y., Wu, M., Feng, Y., Zhou, Z., Chen, L., and Chen, F. 2018. Alterations of oral microbiota distinguish children with autism spectrum disorders from healthy controls. Sci. Rep. 8, 1597.

Robin, X., Turck, N., Hainard, A., Tiberti, N., Lisacek, F., Sanchez, J.C., and Müller, M. 2011. pROC: an open-source package for R and $\mathrm{S}+$ to analyze and compare ROC curves. BMC Bioinformatics $12,77$.

Rozenberg, F. 2013. Acute viral encephalitis. Handb. Clin. Neurol. 112, 1171-1181.

Sampaio-Maia, B., Caldas, I.M., Pereira, M.L., Pérez-Mongiovi, D., and Araujo, R. 2016. The oral microbiome in health and its implication in oral and systemic diseases. Adv. Appl. Microbiol. 97, $171-210$.

Santigli, E., Koller, M., and Klug, B. 2017. Oral biofilm sampling for microbiome analysis in healthy children. J. Vis. Exp. 130, 56320.

Segata, N., Izard, J., Waldron, L., Gevers, D., Miropolsky, L., Garrett,
W.S., and Huttenhower, C. 2011. Metagenomic biomarker discovery and explanation. Genome Biol. 12, R60.

Shoemark, D.K. and Allen, S.J. 2015. The microbiome and disease: reviewing the links between the oral microbiome, aging, and Alzheimer's disease. J. Alzheimers Dis. 43, 725-738.

Thompson, C., Kneen, R., Riordan, A., Kelly, D., and Pollard, A.J. 2012. Encephalitis in children. Arch. Dis. Child. 97, 150-161.

Tuppeny, M. 2013. Viral meningitis and encephalitis. Crit. Care Nurs. Clin. North Am. 25, 363-380.

Venter, M., Zaayman, D., van Niekerk, S., Stivaktas, V., Goolab, S., Weyer, J., Paweska, J.T., and Swanepoel, R. 2014. Macroarray assay for differential diagnosis of meningoencephalitis in southern Africa. J. Clin. Virol. 60, 50-56.

Wang, W. 2013. Pediatrics, 8th edn. People's Medical Publishing House, Beijing, China.

Wright, W.F., Pinto, C.N., Palisoc, K., and Baghli, S. 2019. Viral (aseptic) meningitis: a review. J. Neurol. Sci. 398, 176-183.

Xie, Y., Tan, Y., Chongsuvivatwong, V., Wu, X., Bi, F., Hadler, S.C., Jiraphongsa, C., Sornsrivichai, V., Lin, M., and Quan, Y. 2015. A population-based acute meningitis and encephalitis syndromes surveillance in Guangxi, China, May 2007-June 2012. PLoS ONE 10, $\mathrm{e} 0144366$.

Xing, X.W., Zhang, J.T., Ma, Y.B., He, M.W., Yao, G.E., Wang, W., Qi, X.K., Chen, X.Y., Wu, L., Wang, X.L., et al. 2020. Metagenomic next-generation sequencing for diagnosis of infectious encephalitis and meningitis: a large, prospective case series of 213 patients. Front. Cell. Infect. Microbiol. 10, 88.

Ye, Q., Shao, W.X., Shang, S.Q., Shen, H.Q., Chen, X.J., Tang, Y.M., Yu, Y.L., and Mao, J.H. 2016. Clinical value of assessing cytokine levels for the differential diagnosis of bacterial meningitis in a pediatric population. Medicine $\mathbf{9 5}$, e3222.

Zhang, S., Kong, C., Yang, Y., Cai, S., Li, X., Cai, G., and Ma, Y. 2020. Human oral microbiome dysbiosis as a novel non-invasive biomarker in detection of colorectal cancer. Theranostics 10, 1159511606.

Zhang, L., Yan, J., Ojcius, D.M., Lv, H., Miao, Z., Chen, Y., Zhang, Y., and Yan, J. 2013. Novel and predominant pathogen responsible for the enterovirus-associated encephalitis in eastern China. PLoS ONE 8, e85023.

Zhu, Y., Liu, J., Liu, L., Li, Y., Li, Y., Zhang, W., Lu, G., and Zheng, Y. 2021. Pathogenic characteristics of cerebrospinal fluid among 26 children with viral encephalitis and meningitis. Chin. J. Virol. 37, 583-590. doi: 10. 13242/j.cnki.bingduxuebao.003967. http:// bdxb.cbpt.cnki.net/WKC/WebPublication/paperDigest.aspx? paperID=7f935f77-1cff-4440-b259-5f010ba0aa3f\# 